\title{
Archéopages
}

Archéopages

Archéologie et société

40 | 04-07/2014

Villages

\section{Les villages du Néolithique ancien en Alsace. Un état de la recherche}

\section{Philippe Lefranc}

\section{(2) OpenEdition}

1 Journals

Édition électronique

URL : https://journals.openedition.org/archeopages/588

DOI : 10.4000/archeopages.588

ISSN : 2269-9872

\section{Éditeur}

INRAP - Institut national de recherches archéologiques préventives

\section{Édition imprimée}

Date de publication : 1 juin 2015

Pagination : 18-25

ISSN : 1622-8545

\section{Référence électronique}

Philippe Lefranc, «Les villages du Néolithique ancien en Alsace. Un état de la recherche », Archéopages [En ligne], 40 | 04-07/2014, mis en ligne le 01 juillet 2016, consulté le 22 janvier 2022. URL : http:// journals.openedition.org/archeopages/588; DOI : https://doi.org/10.4000/archeopages.588 


\section{Les villages du Néolithique ancien en Alsace \\ Un état de la recherche}

Philippe Lefranc Inrap, UMR 7044 «Archéologie et histoire ancienne: Méditerranée et Europe»

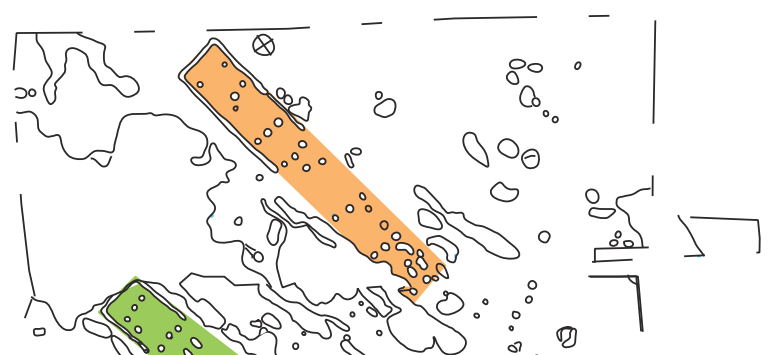

Étapes stylistiques

Rubané ancien

Rubané moyen

Première partie du Rubané récent

Fin du Rubané récent

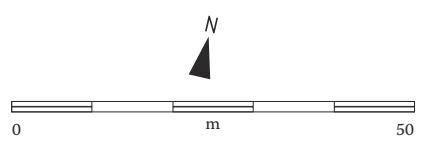

\section{L'habitat de}

Bischoffsheim, étudié

sur une surface de $3 \mathrm{ha}$,

a livré les plans souvent

très bien conservés d'une

quarantaine de bâtiments,

essentiellement des

grandes maisons

tripartites, dont la

construction s'étage sur près de trois siècles. 
L'étude des habitats du Néolithique ancien découverts en Alsace au cours des quinze dernières années est passée au premier rang des objectifs de la recherche régionale. Depuis la fin des années 1990, le corpus des maisons rubanées recensées dans ce secteur géographique a en effet été multiplié par dix, à la faveur notamment des grands décapages préventifs qui ont également permis de mieux appréhender l'organisation interne de ces habitats, ainsi que la question de leur inscription au sein de larges réseaux de sites. Il est donc nécessaire d'ordonner cet important corpus qui fait, depuis 2014, l'objet d'un axe de recherche ${ }^{\mathbf{1}}$. Cette contribution propose un rapide état des connaissances, ainsi que quelques pistes de recherches. Rappelons que la région considérée est occupée par deux groupes régionaux du Rubané se distinguant au niveau des styles céramiques, des gestes funéraires, des systèmes d'élevage et de l'architecture. Ils s'étendent respectivement sur la Basse- et la Haute-Alsace, la frontière stylistique étant située au niveau de la ville de Colmar (Jeunesse, 1995).

Le corpus régional des maisons rubanées s'élève aujourd'hui à environ 150 bâtiments, inégalement répartis entre la Haute et la Basse-Alsace. Le groupe stylistique bas-alsacien est le mieux documenté, avec près d'une centaine de maisons identifiées provenant essentiellement des habitats étudiés à Bischoffsheim «Afua du stade» (Jeunesse, Sainty, 1991; Lefranc et al., 2004; Lefranc, 2007), Entzheim «Les Terres de la Chapelle» (Lefranc et al., 2012), Rosheim «Sainte-Odile/Rittergass » (Jeunesse, Lefranc, 1999; Lefranc, Michler, à paraître), Osthouse (Perrin, 2013) et Kolbsheim (Denaire, 2013). L'inventaire haut-alsacien est moins étoffé, avec à peine une quarantaine de maisons, pour la plupart mises au jour à Sierentz «Sandgrube» et «Tiergarten» (Wolf, 1997; Lefranc, Denaire, 200o) et à Ungersheim «Bioscope» (Châtelet, 2006). Il faut ajouter à ce corpus une quinzaine de bâtiments localisés au niveau de la frontière, à Wettolsheim «Ricoh» (Jeunesse, 1993a) et à Colmar «Route de Rouffach» (Jeunesse, 1993b). Si l'on ne retient dans cet inventaire que les maisons dont l'appartenance à un type de bâtiment peut être précisée, le corpus chute à moins d'une centaine de bâtiments, dont les trois quarts sont localisés en Basse-Alsace. Dans l'ensemble de la région, trois sites seulement ont livré plus de dix maisons : Bischoffsheim (41 maisons), Entzheim (23 maisons) et Sierentz (15 maisons), habitats sur lesquels a été constitué l'essentiel de la documentation se rapportant à l'architecture et à l'organisation spatiale des villages rubanés du sud de la plaine du Rhin supérieur.

\section{L'architecture: trois types de maisons}

En Basse-Alsace, région où l'architecture a pu être documentée pour toute la séquence rubanée, de l'étape ancienne à l'étape finale, les maisons sont en règle générale de plan rectangulaire même si, vers la fin du Rubané récent, apparaissent quelques plans trapézoïdaux évoquant la tradition architecturale haute-alsacienne. À de rares exceptions près, les types rencontrés s'intègrent à la typologie de Modderman (Modderman, 1970) qui distingue les bâtiments tripartites (type 1), les maisons bipartites (type 2) et les maisons réduites au seul module central (type 3).

Les maisons tripartites sont les plus nombreuses (39 occurrences), et ce quelle que soit l'étape stylistique considérée. La variabilité typologique observée entre les bâtiments de type 1 est essentiellement fonction de facteurs chronologiques: on pense notamment à la présence du dispositif en Y ou aux fossés extérieurs (Aussengraben) (Nowicki et al., 1997) rencontrés sur les bâtiments du Rubané ancien, mais également à d'autres caractères architecturaux plus rarement notés tels que l'accroissement de la taille des maisons entre les étapes ancienne et récente. D’une longueur comprise entre 20 et $30 \mathrm{~m}$ pour une largeur oscillant entre 5 et $6 \mathrm{~m}$ au Rubané ancien, elles atteignent très fréquemment, au cours de l'étape récente, $40 \mathrm{~m}$ de long pour une largeur avoisinant $7 \mathrm{~m}$. Il existe également, sur le site de Bischoffsheim [ill. 1], quelques très longues maisons de plus de $45 \mathrm{~m}$ datées des étapes ancienne et moyenne; il s'agit là de bâtiments relativement étroits, construits sur les mêmes modules que les maisons plus modestes, mais qui se particularisent par un fort développement de la partie arrière. Les très longues maisons sont également attestées lors de l'étape récente, notamment à Kolbsheim «Vogeseblick» où un bâtiment de plus de $56 \mathrm{~m}$ de long, caractérisé par un allongement des parties arrière et centrale, a récemment été étudié (Denaire, 2013).

Les maisons bipartites de type 2 ( 29 occurrences) sont des édifices de taille modeste, dépourvus de partie avant, qui n'excèdent pas 10 à $15 \mathrm{~m}$ de longueur. Leur partie arrière est peu développée, le plus souvent limitée à une unique travée selon un schéma également attesté en Haute-Alsace et dans le Rubané du Nord-Ouest. Il est possible que, vers la fin de l'étape récente, notamment à Rosheim «Sainte-Odile/Rittergass » (Lefranc, Michler, à paraître), apparaissent en Basse-Alsace des maisons longues bipartites comparables à celles rencontrées sur les habitats du Bassin parisien, par exemple à Berry-au-Bac «Le Chemin de la Pêcherie» (Ilett, Plateaux, 1995), Cuiry-lès-Chaudardes (Ilett et al., 1980 ; Ilett, Hachem, 1987), Bucy-le-Long «La Fosselle» (Hachem et al., 1998) ou Mennevile «Derrière le Village» (Farruggia et al., 1996). Ces bâtiments bipartites, qui peuvent dépasser $25 \mathrm{~m}$ de longueur, se distinguent de leurs homologues alsaciens par leur taille importante qui dérive essentiellement d'un fort développement de la partie arrière pouvant compter de deux à trois travées.

Enfin, les bâtiments de type 3 demeurent rares (7 occurrences) et souvent difficile à caractériser. Comme il est de règle en Basse-Alsace, tous les bâtiments sont orientés dans un quadrant nord- le groupe Préhistoire de

l'UMR 7044 Archimède

(Université de Strasbourg). 
2. Les groupes bas- et haut-alsaciens du Rubané

se distinguent

notamment par

l'orientation des

bâtiments, d'axe nord-

ouest/sud-est en Basse-

Alsace (en bleu) et proche

de l'axe est-ouest en

Haute-Alsace (en orange)

Dans le secteur de la

frontière (en vert), les

orientations des maisons

recouvrent celles de la

Basse-Alsace et du nord

de la Haute-Alsace.
3. Les neuf maisons de ce

secteur du site de

Bischoffsheim (Bas-

Rhin), que l'on peut

attribuer à cinq stades

stylistiques successifs,

illustrent le système du

Hofplatz caractérisé par

le déplacement d'une

même maisonnée au sein

d'un espace prédéfini. On

notera que l'espace de la

ferme peut être

simultanément occupé

par deux bâtiments de

types différents (aux

stades IIb et III).
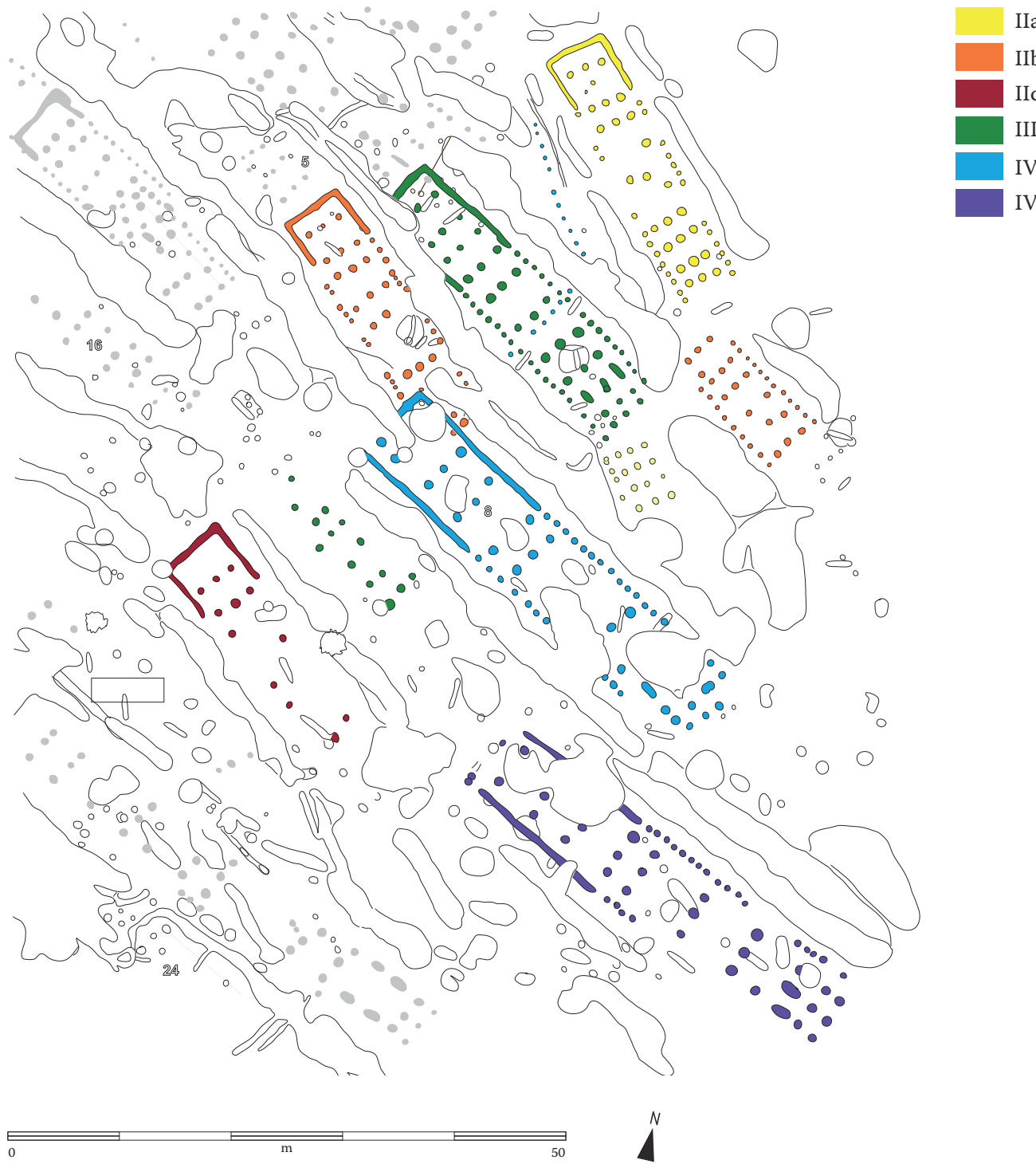
ouest. L'analyse des maisons bien datées de Bischoffsheim a montré qu'à l'intérieur de ce quadrant, les bâtiments s'organisaient en groupes d'orientation étroitement corrélés aux étapes stylistiques et marqués par un glissement progressif vers l'axe est-ouest entre l'étape ancienne et l'étape récente (Lefranc, 2007). Ce phénomène s'observe également à Entzheim «Les Terres de la Chapelle». Au sein du groupe stylistique haut-alsacien, seul l'habitat de Sierentz «Sandgrube», bien publié, a livré des plans de maisons suffisamment conservés pour se prêter à l'analyse. Les variations architecturales à caractère chronologique décelées en Basse-Alsace n'apparaissent pas en Haute-Alsace, la chronologie des maisons fouillées ne recouvrant que les étapes récente et finale de la séquence régionale. À l'exception du plan trapézoïdal, adopté dès la charnière Rubané moyen/récent (Jeunesse et al., 2007) et d'une orientation proche de l'axe est-ouest, les bâtiments ne diffèrent pas fondamentalement de leurs homologues du Rubané récent de Basse-Alsace, qu'il s'agisse des dimensions ou des types architecturaux rencontrés. On recense, sur les habitats du groupe de Haute-Alsace et sur les habitats de la frontière, 14. maisons de type 1, quatre maisons de type 2 et une seule maison de type 3. Les orientations enregistrées à Sierentz, habitat le plus méridional aujourd'hui documenté dans le sud de la plaine d'Alsace, suivent des axes ouest/est ou ouest-sudouest-est-nord-est. À Ungersheim, village situé plus au nord, à mi-chemin entre Sierentz et le secteur de la frontière stylistique (Châtelet, 2006), les orientations se répartissent de part et d'autre de l'axe est-ouest. Enfin, on doit noter que dans le secteur de la frontière, documenté par le site à occupation mixte de Wettolsheim «Ricoh», l'éventail des orientations recoupe à la fois celles enregistrées sur les maisons du Rubané récent de Basse-Alsace et celles qui caractérisent l'habitat d'Ungersheim [ill. 2].

\section{L'organisation et l'évolution de l'espace}

Le modèle d'organisation interne des villages rubanés qui domine l'histoire de la recherche depuis son élaboration par l'équipe du plateau d'Aldenhoven est celui du Hofplatz (Stehli, 1989). Selon ce schéma, les villages sont constitués d'unités élémentaires d'habitation regroupant la maison elle-même, les fosses latérales ainsi qu'un nombre variable de fosses satellites. Cette unité atteignant une extension d'environ $25 \mathrm{~m}$ de rayon autour de la maison, la distance minimale entre deux bâtiments contemporains est donc en théorie de l'ordre d'une cinquantaine de mètres. Le Hofplatz correspond à la parcelle sur laquelle se succèdent plusieurs fermes, régulièrement reconstruites par plusieurs générations d'un même groupe social (Lüning, 1998). Ces Hofplätze peuvent développer des traditions céramiques propres, présenter des indices de spécialisation ou montrer des écarts de richesse significatifs. La stricte application de ce modèle au village de Bischoffsheim a permis de distinguer six ensembles - plus ou moins satisfaisants regroupant en moyenne huit maisons diachrones pouvant être identifiées aux bâtiments successifs régulièrement reconstruits par une même maisonnée au sein d'un même périmètre et sur une durée d'environ trois siècles. Les ensembles les plus convaincants, rassemblant des maisons bien datées, montrent une succession de bâtiments recouvrant sans hiatus apparent toute la durée du Rubané de Basse-Alsace entre les stade ancien B et récent IVb (Lefranc et al., 2004) [ill. 3]. Les maisons semblent s'organiser de manière aléatoire au sein de ces ensembles, mais, dans deux cas au moins, il est possible de lire en filigrane un semblant d'organisation en rangée. Ce type d'organisation a souvent été noté sur l'ensemble du domaine rubané, par exemple à Frimmersdorf en Rhénanie (Weiner et al., 2010), à Ulm-Eggingen (Kind, 1989), à Cuiry-lès-Chaudardes (Coudart, 1998) ou à Sierentz (Wolf, 1997). En s'appuyant sur certains de ces plans, Oliver Rück s'est récemment attaché à déconstruire le modèle du Hofplatz et a proposé une lecture radicalement différente de l'organisation des villages rubanés qui seraient formés de rangées de maisons parallèles, en grande partie contemporaines et situées le long de rues ou autour de places dans le cadre d'une occupation de l'espace villageois dûment planifiée (Rück, 2011, 2014). Les quelques exemples alsaciens analysables nous obligent à nous inscrire en faux contre cette reconstruction qui semble faire très peu de cas de la chronologie. Il s'agit en premier lieu du village d'Entzheim «Les Terres de la Chapelle», récemment étudié (Lefranc et $a l .$, 2012) et qui offre, malgré une érosion assez marquée, un bel exemple de maisons construites sur plusieurs rangées étagées [ill. 4]. Or, l'abondante céramique décorée recueillie dans les fosses latérales associées aux maisons montre, sans ambiguïté possible, que les bâtiments frappés d'alignement sont très probablement tous diachrones. Le cas de Sierentz est également éclairant: ce vaste village s'étendant sur une dizaine d'hectares n'a malheureusement bénéficié que de fouilles portant sur des surfaces relativement réduites (Wolf, 1997 ; Lefranc, Denaire, 200o), mais plusieurs ensembles de maisons adjacentes et aux façades parfaitement alignées ont été observés [ill. 5]. Comme à Entzheim, l'étude de la céramique décorée a permis de montrer que les maisons constitutives de chaque ensemble relevaient de trois à quatre stades stylistiques successifs et que ces rangées résultaient probablement du déplacement d'une même maisonnée au sein d'un espace prédéfini (Lefranc, 2001). Il convient enfin de rappeler que l'organisation des maisons en rangée, déjà signalée sur le site de Langweiler 9 (Hofplatz $\mathrm{n}^{\circ} 3$ ) a joué un rôle important dans l'élaboration du modèle des Hofplätze (Kuper et al., 1977; Lüning, 1998). 
4. Le village rubané

d'Entzheim,

malheureusement très

érodé, a livré un exemple

de bâtiments organisés en

rangées horizontales.

Cette configuration

pourrait résulter d'une

contrainte imposée par un

quadrillage de l'espace

villageois, une sorte de

parcellaire que l'on peut

également restituer sur le

site de Bischoffsheim.

5. Le site de Sierentz, très

partiellement fouillé,

offre un exemple peu

discutable d'organisation

en rangées. L'analyse de la

céramique décorée a

permis d'établir que

les bâtiments frappés

d'alignements

appartenaient très

probablement à des

stades stylistiques

successifs.
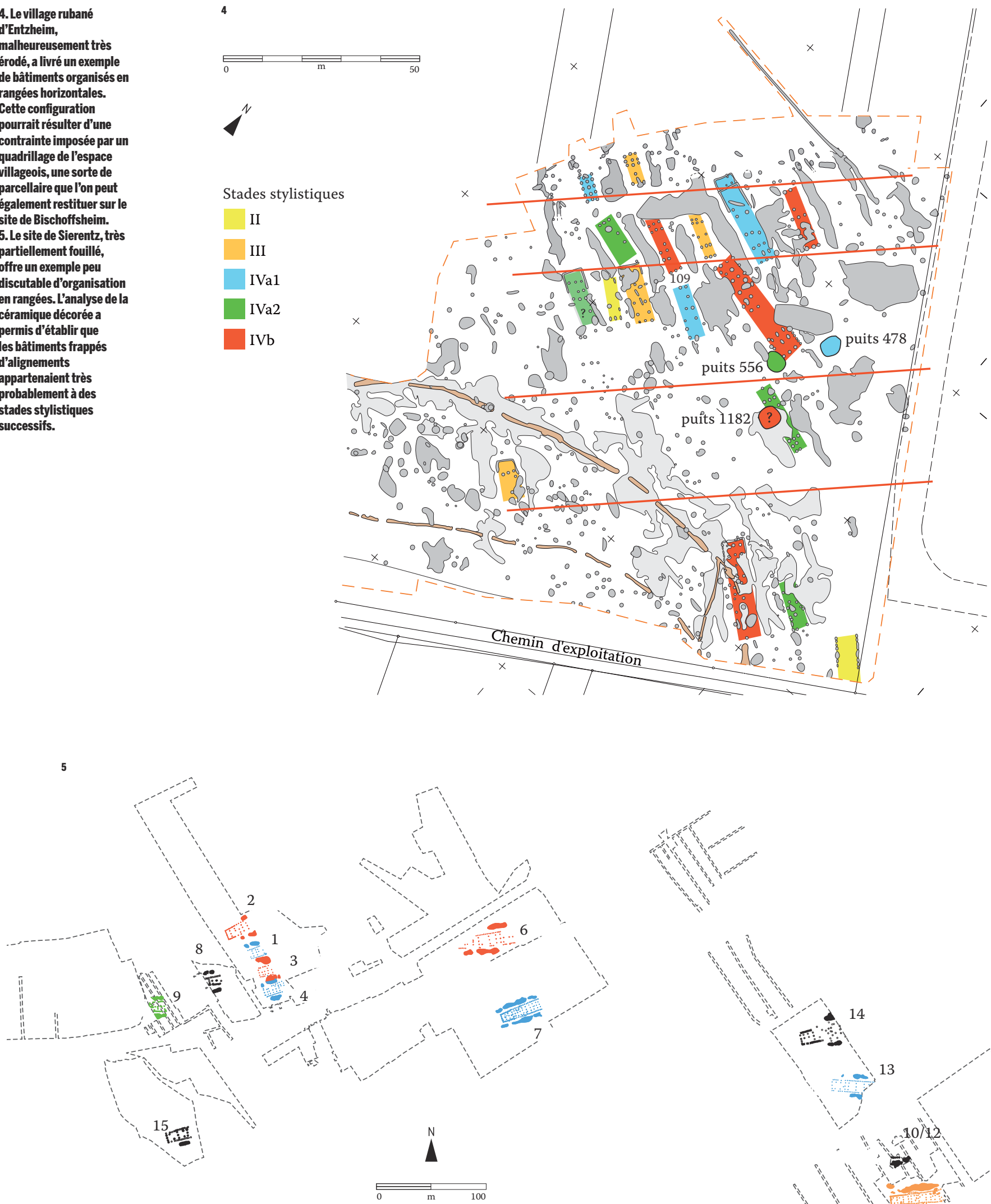

--- Limite de décapage

Rubané récent. Stade non déterminé

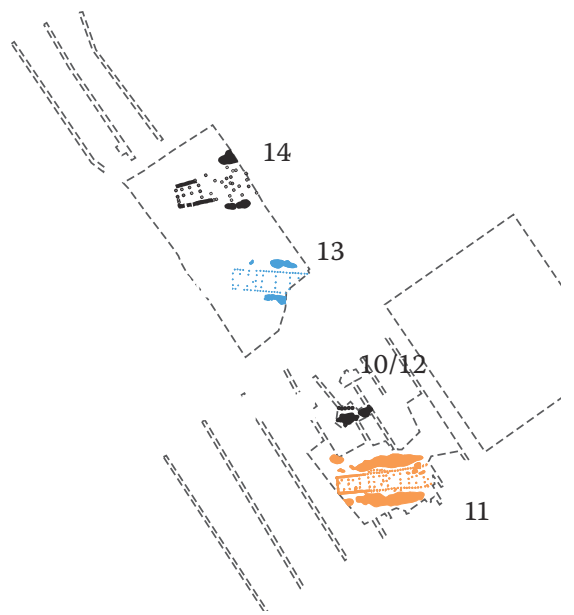

Transition moyen/récent A

Rubané récent $\mathrm{A}$

Rubané récent $B$

Rubané final 
L'analyse de l'organisation interne des villages alsaciens se conforme bien à ce modèle, qui peut être accepté dans son principe; l'existence de groupes composés de maisons diachrones, édifiées par un même segment social tout au long de l'occupation du village, ne peut en effet être sérieusement remise en cause. Il nous semble cependant que le modèle puisse être en partie amendé: à Bischoffsheim comme à Entzheim, nous avons pu observer que des maisons potentiellement contemporaines présentaient une façade et un pignon arrière très rapprochés, configuration résultant très probablement d'une implantation des maisons à l'intérieur d'un espace quadrillé délimitant des parcelles rectangulaires, comme on peut en observer à Entzheim et sans doute à Sierentz; ici, l'espace utile de la ferme ne s'étend pas tout autour de la maison - comme le suppose le modèle classique -, mais se développe uniquement de part et d'autre des longs côtés des bâtiments, proposition qui pourrait impliquer un réexamen de nombre de reconstitutions de Hofplätze rencontrées dans la littérature. Selon le modèle d'occupation accepté ici, le nombre de maisons contemporaines composant le village de Bischoffsheim s'élèvent à six pour la partie fouillée et aux alentours d'une douzaine au maximum, si l'on estime que seule une moitié du site a été reconnue. Les diverses estimations du nombre d'individus constituant la maisonnée rubanée, bien que fondées sur des modèles ethnographiques, restent théoriques et très difficile à manipuler (Dubouloz, 2008). Tout au plus peut-on, en prenant en compte la moyenne parfois avancée de 13 habitants par maison (ibid.), retenir pour la partie fouillée du site un ordre de grandeur situé autour d'une centaine de personnes. Il ne semble pas exister, à l'intérieur des villages du Rubané alsacien, d'espaces demeurés vides de vestiges et que l'on pourrait assimiler à des places de rassemblement communautaire. Les seules structures collectives connues sont les puits à eau que nous avons pu étudier à Ittenheim (Lefranc et $a l ., 2010$ ) et à Entzheim (Lefranc et al., 2012), et qui sont installés puis régulièrement reconstruits au sein d'un même périmètre localisé à la périphérie immédiate de l'espace villageois et pouvant faire office de place de rassemblement. L'espace villageois peut éventuellement être délimité par un fossé continu peu profond, à fond plat et à profil en cuvette. Deux enceintes de ce type, attribuées à l'étape ancienne, sont attestées à Colmar «Route de Rouffach » et à Wettolsheim « Ricoh» (Jeunesse, 1993a et b). Une troisième enceinte, un peu plus récente (transition Rubané moyen/récent), mais de morphologie identique, a été observée à Ittenheim «Complexe sportif» (Lefranc et al., 2010).

Les réseaux d'habitats : capitales microrégionales et sites satellites Le nombre des habitats fouillés sur de vastes surfaces est encore trop restreint pour nous permettre d'appréhender avec une précision suffisante la question de la hiérarchisation des villages du Rubané alsacien. Cependant, un habitat au moins peut être attribué, avec une forte probabilité, à la catégorie des «sites centraux» tels qu'ils ont été définis par Jürgen Kneipp (1995) et Jens Lüning (1998) en Hesse et en Rhénanie. L'assimilation de l'habitat de Rosheim «SainteOdile», partiellement fouillé (Jeunesse, Lefranc, 1999; Lefranc, Michler, à paraître), à une capitale microrégionale assurant des fonctions cérémonielles et économiques particulières se fonde sur la présence d'une enceinte à pseudofossé, monument à probable vocation cérémonielle fédérant les membres d'une même communauté; sur un mobilier riche et diversifié traduisant des échanges à longue distance, illustrés notamment par des vases importés des régions du Neckar, de la Hesse et du Hegau; et enfin sur sa très probable fonction d'importateur et de redistributeur de matières premières. Le site, et c'est une exception pour l'ensemble des habitats de Basse-Alsace, a en effet livré un nombre élevé d'artéfacts en roches tenaces, sous forme d'ébauches et de pièces finies, laissant envisager un contrôle de la diffusion des roches cristallines et des diabases vosgiennes dans la région proche (Mauvilly, 2001). La plupart des découvertes de «Sainte-Odile» sont attribuées aux étapes récente et finale, mais on peut envisager que ce statut de capitale microrégionale ait déjà été celui de l'habitat du Rubané ancien qui se développe à l'est de ce village: il s'agit en effet du seul secteur de Basse-Alsace ayant livré des fragments de statuettes rubanées (Thévenin, Munger, 1971; Lefranc, 2006), objets particulièrement nombreux sur les sites du Rubané ancien assurant des fonctions cérémonielles (Schade-Lindig, 2002). Il est difficile d'estimer l'étendue du groupement de sites dont le site central de Rosheim constituerait le centre symbolique. La répartition des habitats rubanés de l'étape récente au sud de la vallée de la Bruche montre une concentration d'une douzaine de villages ou de hameaux s'égrainant de part et d'autre du ruisseau du Rosenmeer, sur les communes de Rosheim, Bischoffsheim, Innenheim, Krautergersheim, Niedernai et Altorf, et qui pourraient constituer une même unité territoriale [ill. 6]. Selon cette hypothèse, ce groupement de sites s'inscrirait dans un cercle d'environ $10 \mathrm{~km}$ de diamètre. La plupart de ces habitats, possibles satellites de Rosheim, sont malheureusement assez peu documentés, à l'exception notable du village de Bischoffsheim qui, en dépit d'un nombre élevé de maisons bien conservées, n'a livré qu'un mobilier des plus communs, de très rares outils polis et, il faut le souligner, un important corpus céramique ne comptant qu'un seul tesson décoré dans un style exogène - et encore s'agit-il d'une importation haute-alsacienne. Le contraste avec Rosheim 


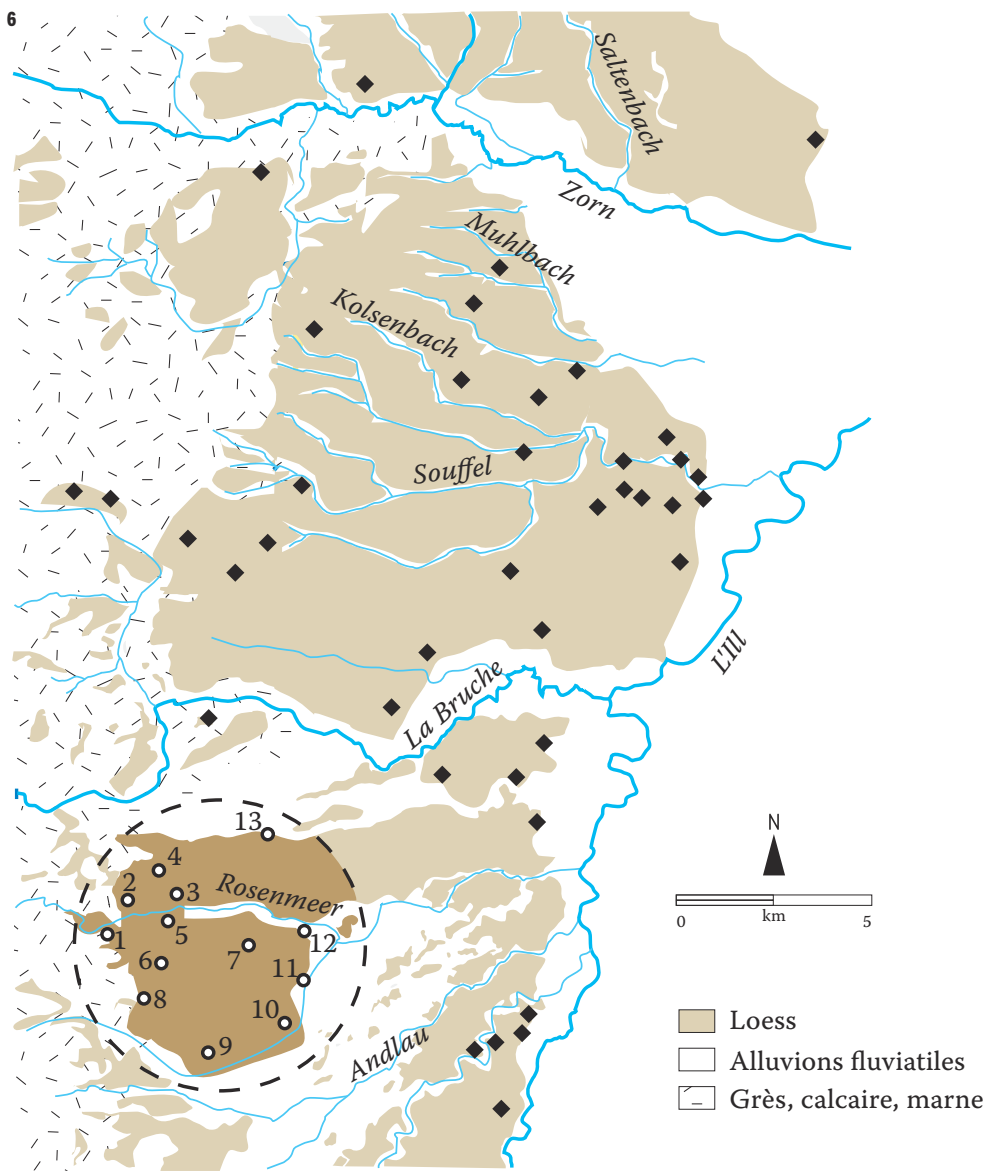

1. Rosheim «Sainte-Odile/Rittergass»

2. Rosheim "Grün»

3. Rosheim «Helmbacher/Rosenmeer»

4. Rosheim «Grunenwegfeld»

5. Rosheim «Bischenabwand»

6. Bischoffsheim «Afua du stade»

7. Bischoffsheim «Bruehling»

6. La carte de répartition du Rubané de BasseAlsace fait apparaître plusieurs concentrations de sites, dont une, bien documentée, pourrait correspondre à un groupement d'habitats dépendant du site central de Rosheim « SainteOdile I.
8. Obernai «Im Thal» 9. Niedernai «Foegel» 10. Krautergersheim «Sud du Village» 11. Krautergersheim «Rue de l'École» 12. Innenheim «Litten»

13. Altorf
«Sainte-Odile» est des plus marqués et vient étayer l'hypothèse d'un village satellite inféodé à ce site central distant d'à peine $3 \mathrm{~km}$.

\section{La place des morts : nécropoles, sépultures en habitat et dépôts atypiques}

Les liens entre habitats et nécropoles sont bien documentés par les sites bas-rhinois de Vendenheim «Le Haut du Coteau» (Jeunesse et al., 2002) et d'Ingenheim «Bannenberg» (Lefranc et al., 2014) où ont été étudiés des ensembles funéraires attribués au Rubané récent (respectivement 100 et 36 sépultures) localisés à une cinquantaine de mètres d'un habitat contemporain. Nous ignorons cependant si l'habitat proche est le seul pourvoyeur du cimetière ou si ce dernier accueille les membres choisis de diverses communautés. Les restes humains dispersés à l'intérieur de l'espace villageois apparaissent sur moins d'une demidouzaine de sites, à Bischoffsheim (Jeunesse, Sainty, 1991; Lefranc, 2007), Ittenheim «Complexe sportif» (Lefranc et al., 2010), Colmar « Route de Rouffach» (Jeunesse, 1993b), Rouffach «Gallbühl» (Sainty et al., 1984) et Entzheim «Les Terres de la Chapelle», ce dernier habitat se démarquant par un nombre élevé de 15 inhumations (Lefranc et al., 2012). Il faut souligner, en Basse-Alsace, la fréquence de ce type d'inhumation, présent sur tous les sites ayant fait l'objet de vastes décapages. Dans quelques cas seulement, les corps ont été déposés dans des fosses de construction ou des fosses de plan circulaire de type Kesselgruben détournées de leur fonction primaire, mais l'essentiel du corpus est constitué d'inhumations régulières en tombe plate. Au sein de ces dernières, on observe en Alsace comme dans le BadeWurtemberg (Orschiedt, 1997) une proportion d'individus immatures ( $55 \%$ du corpus) bien supérieure à celle enregistrée dans les nécropoles régionales (22\% à Vendenheim, Jeunesse et al., 2002), ainsi qu'une très faible proportion de tombes bénéficiant de dépôts de mobiliers funéraires ( $7 \%$ des tombes en habitat contre $45 \%$ des tombes de Vendenheim et $47 \%$ des tombes d'Ingenheim; Lefranc et al., 2014).

Outre ces sépultures régulières, les habitats rubanés livrent également des restes humains ayant fait l'objet de manipulations périmortem, à l'image de la probable tête coupée mise au jour dans une fosse d'extraction d'Ittenheim (Lefranc et al., 2010) et du corps mutilé inhumé sur le tracé de l'enceinte du même site (Lefranc, Boës, 2009). Enfin, il faut insister sur l'existence de corps inhumés dans des fosses d'habitat, dans des positions irrégulières trahissant sans doute l'absence de gestes funéraires. Cette catégorie, encore peu signalée dans les contextes du Néolithique ancien régional, est notamment illustrée par deux individus gisant sur le ventre et en position désordonnée à Kolbsheim (Denaire, 2013) et à Entzheim «Les Terres de la Chapelle», ainsi que par un jeune enfant, mis au jour sur ce dernier site, inhumé sur le dos, jambes repliées sur la poitrine et accompagné de deux chevilles osseuses d'Aurochs (Lefranc et al., 2012).

Le bilan régional sur l'habitat du Rubané en Alsace entrepris par l'UMR 7044 permettra sans doute, dans un avenir proche et en s'appuyant sur un important corpus de sites, de préciser ou d'amender l'état des connaissances rapidement esquissé ici, notamment sur les thèmes de la variabilité architecturale, de la chronologie des habitats, des modèles d'organisation spatiale ou, thème non abordé dans cette contribution, sur la structure sociale des communautés villageoises telle qu'on peut la saisir à travers les plans des villages et la répartition des différentes catégories de mobilier. 
Jeunesse C., Wolf J.-J., LefranC P.,

SChaltenbrand K., 2007, «Rubané du sud-ouest et maison trapéziforme: l'exemple de la maison 11 de Sierentz (Haut-Rhin) », in Agogué O., Leroy D., VerJux C. (DIR.), Camps, enceintes et structures d'habitat néolithique en France septentrionale, Actes du XXIV Colloque interrégional sur le Néolithique, Orléans, 19-21 novembre 1999, Tours, Feracf (coll. Suppl. à la Revue archéologique du centre de la France), p. 39-54.

KIND C.-J., 1989, Ulm-Eggingen. Die Ausgrabungen. 1982 bis 1985 in der bandkeramischen Siedlung und der mittelalterlichen Wüstung, Stuttgart, Konrad Theiss Verlag (coll. Forschungen und Berichte zur Vor- und Frühgeschichte in Baden-Württemberg, 34), $500 \mathrm{p}$.

KNEIPP J., 1995, «Frühbäuerliche Siedlungsverbände am Diemel, Esse und Unterer Fulda. Gedanken zur sozialen und wirtschaftlichen Struktur vor 7000 Jahren ", Zeitschrift des Vereins für Hessische Geschichte und Landeskunde, 100, p. 1-19.

Kuper R., Löhr H., LÜNING J., Stehli P., ZimMERMANN A., 1977, «Der bandkeramische Siedlungsplatz Langweiler 9, Gemeinde Aldenhoven Kreis Düren. Beiträge zu neolitischen Besiedlung der Aldenhovener Platte II », Rheinische Ausgrabungen, 18, 3 vol.

LEFRANC P., 2001, «La fin de la séquence rubanée en Haute-Alsace (Rubané récent et final) vue à travers l'évolution de la céramique décorée du site de Sierentz-Sandgrube et Tiergarten (Haut-Rhin)», Bulletin de la Société préhistorique française, 98 , p. $627-646$.

LEFRANC P., 2006, «Un nouveau fragment de statuette du Rubané ancien à Rosheim (Bas-Rhin)», in Duhamel P. (DIR.), Impacts interculturels au Néolithique moyen, Actes du XXV Colloque interrégional sur le Néolithique, Dijon, 20-21 octobre 2001, Dijon, Société archéologique de l'Est (coll. Suppl. à la Revue archéologique de l'Est, 25), p. 253-259.

LEFRANC P., 2007, «L'habitat rubané de Bischoffsheim (Bas-Rhin), premiers résultats », Archaeologia Mosellana, 7, p. 9-21.

LEFRANC P., BOËs E., 2009, «Les restes humains manipulés du site rubané d'Ittenheim "Complexe sportif” (Bas-Rhin)», in ZeEb-LANZ A. (ED.), Internationale Fachtagung «KrisenKulturwandel - Kontinuitäten: Zum Ende der Bandkeramik in Mitteleuropa » vom 14.06. 17.06.2007 in Herxheim bei Landau (RheinlandPfalz), Rhaden, Marie Leidorf (coll. Internationale Archäologie, Arbeitsgemeinschaft, Symposium, Tagung, Kongress, 10), p. 117-211.

LefranC P., Denaire A., 20oo, « Deux nouvelles maisons du Néolithique ancien rubané et une fosse de la culture de Grossgartach à Sierentz "Tiergarten", Haut-Rhin », Cahiers de l'Association pour la promotion de la recherche archéologique en Alsace, 16, p. 17-36.

LEFRANC P, MICHLER M., à paraître, «Une nouvelle maison du Rubané récent sur le "site central" de Rosheim "Rittergass"/"Sainte-Odile" (Bas-Rhin) », article soumis à la Revue archéologique de l'Est.

Lefranc P., Denaire A., Boës E., 2010, «Le site Néolithique ancien et moyen d'Ittenheim (BasRhin) », Revue archéologique de l'Est, 59, p. 65-97.

LeFranc P., Alix G., Latron F., 2014, «La nécropole Rubané récent d'Ingenheim "Bannenberg", BasRhin », in Lefranc P., Denaire A., Jeunesse C. (DIR.), Données récentes sur les pratiques funéraires néolithiques de la plaine du Rhin supérieur, Oxford, British Archeological Reports (coll. BAR International Series, 2633), p. 5-58.

Lefranc P., BAKaj B., Robert F., Zehner M., 2004, Bischoffsheim "Afua du Stade», Document final de synthèse de fouilles d'archéologie préventive, Strasbourg, SRA Alsace/Antea, 2 vol.

Lefranc P., Alix G., Chenal F., Schneider N., 2012, Entzheim, Bas-Rhin, In der Kappell Z.A. «Les Terres de la Chapelle $2 »$. Village rubané, enceinte et inhumations de la fin du $V^{*}$ millénaire et habitat Michelsberg, Document final de synthèse, Inrap Grand-Est sud, 3 vol.
LÜNING J., 1998, «L'organisation régionale des habitats rubanés : sites centraux et sites secondaires (groupements de sites) ", in CAUwE N., VAN BERG P.-L. (DIR.), Organisation néolithique de l'espace en Europe du Nord-Ouest, Actes du XXIII ${ }^{e}$ Colloque interrégional sur le Néolithique, Bruxelles, 24-26 octobre 1997, Bruxelles, Société royale belge (coll. Anthropologie et préhistoire, 109), p. 163-185.

MaUvilly M., 2001, «Le mobilier lithique du site rubané de Rosheim "Lotissement Sainte-Odile" (Bas-Rhin) », Cahiers de l'Association pour la promotion de la recherche archéologique en Alsace, 17, p. 327-358.

ModDerman P.-J. R., 1970, «Linearbandkeramik aus Elsloo und Stein », Analecta Praehistorica Leidensia, 3, $218 \mathrm{p}$

Nowicki P., SAinty J., Jeunesse C., 1997, «Un nouvel habitat du Néolithique ancien et moyen à Achenheim (Bas-Rhin)», Cahiers de l'Association pour la promotion de la recherche archéologique en Alsace, 13, p. 17-24.

OrSChIEDT J., 1997, «Sépultures rubanées en habitat dans le Bade-Wurtemberg. Études archéologiques et anthropologiques », in JEUNESSE C. (DIR.), Le Néolithique danubien et ses marges entre Rhin et Seine, Actes du XXII Colloque interrégional sur le Néolithique, Strasbourg, 27-29 octobre 1995, Strasbourg, Cahiers de l'Association pour la promotion de la recherche archéologique en Alsace (coll. Suppl. aux Cahiers de l'Association pour la promotion de la recherche archéologique en Alsace, 3), p. 57-63.

Perrin B., 2013, Osthouse «Kleinfeld» (Bas-Rhin, Alsace), Rapport de fouilles préventives, Strasbourg, SRA Alsace, 194 p., 61 pl. et annexes.

RüCK O., 2011, « Le village rubané : rangées de maisons et espaces vierges ouvrent de nouvelles perspectives sur le développement et l'organisation de l'habitat », in Hauzeur A., JAdIN I., Jungels C. (DIR.), 5000 ans av. J.-C. : la grande migration? Le Néolithique ancien dans la collection Louis Éloy, Bruxelles, Institut du patrimoine wallon, p. 35-41.

RüCK O., 2014, «From Yard to House Row: the Bandkeramik Village - Layouts in Rows and Feature-Free Areas Provide a New View on Settlement Structure », in Hamon C., Allard P., ILETT M. (ED.), The Domestic Space in LBK Settlements, Rhaden, Marie Leidorf (coll. Internationale Archäologie, Arbeitsgemeinschaft, Symposium, Tagung, Kongress, 17), p. 201-230.

Sainty J., Thevenin A., Thevenin C., Piningre J.-F., 1984, «Le site rubané récent de Rouffach "Gallbühl" (Haut-Rhin)», Revue archéologique de l'Est, 35-1.2, p. 3-39.

SCHADE-Lindig S., 2002, «Idol- und Sonderfunde der bandkeramischen Siedlung von Bad-NauheimNieder-Mörlen "Auf dem Hempler" (Wetteraukreiss) », Germania, 8o, p. 47-114

STEHLI P., 1989, «Merzbachtal-Umwelt und Geschichte einer bandkeramischen Siedlungskammer », Germania, 67-1, p. 51-76.

Thévenin A., Munger C., 1971, «Découverte d'un fragment de statuette néolithique à Rosheim », Bulletin de la Société préhistorique française, 68 , p. 533-539.

Weiner J., Biemann E., Cziesla E., Gaitzsch W., GeIlenbrüGge U., Heinen M., Ibeling T. MüNCH U., 2010, «Frühe Ackerbauern im Rheinland. Was gibt es neues seit 2005 ?», in Otten T., Hellenkemper H., Kunow J., RIND M. (EDS.), Fundgeschichten. Archäologie in Nordrhein-Westfalen, Köln, RömischGermanisches Museum, p. 59-64.

WoLF J.-J., 1997, «Sierentz (Haut-Rhin). Un habitat rubané de Haute-Alsace», in JEUNESSE C. (DIR.), Le Néolithique danubien et ses marges entre Rhin et Seine, Actes du XXII Colloque interrégional sur le Néolithique, Strasbourg, 27-29 octobre 1995, Strasbourg, Cahiers de l'Association pour la promotion de la recherche archéologique en Alsace (coll. Suppl. aux Cahiers de l'Association pour la promotion de la recherche archéologique en Alsace, 3), p. 407-410. T., SCHNEIKERT F., SIDERA I., 2002, du Néolithique ancien, Document final de synthèse, Strasbourg, Inrap/SRA Alsace, 3 vol. 